\title{
MODELOS PAISAJÍSTICOS DEL REBOLLAR EN EXTREMADURA
}

\author{
Felipe Leco Berrocal*
}

\begin{abstract}
RESUMEN
La degradación actual de los bosques de quercíneas en Extremadura es signo inequívoco de un abandono e inadecuada gestión sobre los mismos. Incendios, talas, repoblación con especies alóctonas,..., son algunas formas que han llevado a la degradación de la vegetación climácica del rebollar. La situación actual de fosilización de estos bosques hace aún más frágil su conservación y desarrollo.
\end{abstract}

Palabras clave: degradación, vegetación climácica, variabilidad climática, localización, distribución.

\begin{abstract}
The present degradation of Quercus forest in Extremadura is clearly the result of their abandonment and inadequate management. Forest fires, clearing, afforestation of allochthonous species, are some of the ways which have lead to the degradation of the vegetation dynamics of Quercus pyrenaica. The present fossilization of the forest makes their conservation and development more fragile.
\end{abstract}

Key words: degradation, dynamic vegetation, variability of climate, localization, distribution.

\section{Sobre los aspectos físico-ecológicos del rebollo}

El rebollar es un bosque típico del piso mesomediterráneo subhúmedo a húmedo y su distribución, que evidentemente no es homogénea, depende en gran medida de tres grupos de factores íntimamente ligados con las exigencias y aprovechamientos de esta especie: exigencias edáficas, climáticas y antrópicas.

Se desarrolla generalmente sobre suelos profundos bastante ricos en materia orgánica del tipo tierra parda húmeda y subhúmeda pero, a veces, nos los encontramos sobre suelos

* Dpto. de Geografía y O.T. Universidad de Extremadura. Cáceres. 
sueltos y limosos más llanos y formando otro tipo de formaciones o asociaciones distintas de las que habitualmente aparece.

Es propio de esta especie localizarla en umbrías frescas y muy húmedas por debajo de los $1.000 \mathrm{~m}$. de altitud, encontrándose muy bien desarrollado en los subsectores HurdanoZezerense, Oretano y Marianense (LADERO ÁLVAREZ, 1987).

Una característica particular que lo distingue de otras quercíneas representativas, como la encina (Quercus rotundifolia) y el alcornoque (Quercus suber), es que se trata de una especie de hoja caducifolia.

Los rebollares en general son menos abundantes y ricos en especies subarbustivas y herbáceas que los bosques esclerófilos, aunque suele ir acompañado de un sotobosque de arbustos y vegetación herbácea que aprovechan de manera evidente toda la luz que les llega durante los meses primaverales, cuando aún los rebollos están desnudos de hoja, para desarrollarse y completar su ciclo anual.

Cabría señalar que los pastizales procedentes de áreas inicialmente pobladas por el rebollar son pastizales silíceos con suelos normalmente pobres (MESÓN GARCÍA et al., 1985).

Es una especie que necesita tanto de abundantes precipitaciones (más de $800 \mathrm{~mm}$. anuales), como de una importante humedad asociada, esto significa que su localización geográfica se encuentra muy determinada. De este modo, el rebollar se desarrolla en los sistemas montañosos de la región donde las características edafoclimáticas le permiten un crecimiento fácil.

El último y principal factor de su localización actual es el hombre. La influencia antrópica ha significado para el bosque de rebollos la desaparición total en muchas áreas de Extremadura donde alcanzaba su máximo desarrollo (Sierra de Gata y Las Hurdes, esencialmente), en definitiva, el equilibrio ecológico de estas áreas ha sido profundamente modificado por el hombre (ALBENTOSA, 1982).

Cuando el hombre interviene en la degradación del bosque de rebollos los objetivos que le mueven a ello suelen ser o aprovechar sus maderas y leñas, para lo que suele mantener un estado más o menos arbolado; o utilizar los pastos para alimentar a su ganado (MESÓN GARCÍA et al., 1985).

Una vez sucedido esto todas las funciones esenciales que desempeña el arbolado de Quercus pyrenaica $W$. quedan aniquiladas; protección frente al impacto de la lluvia, escorrentía, sujeción de laderas, bombeo de nutrientes desde las capas profundas del perfil, deterioro de la estructura física y química del suelo (acidificación, reducción de la permeabilidad y reducción de la capacidad de retención de agua,...).

Teniendo en cuenta que el número de términos municipales extremeños tipificados como de montaña (LECO, 1995) ascienden a 86 (un 23,5\% sobre el total regional), sólo detectamos el desarrollo de esta especie en 30 términos que suponen un total de 233.394 has. (un $42,46 \%$ sobre el total de la superficie ocupada por la montaña).

Otros municipios, dentro de la media montaña extremeña, también cuentan con importantes superficies de rebollo, tal es el caso de Tejeda de Tiétar (1.304,8 has.) y Jarandilla de la Vera (1.120,3 has.). También destacan otros términos municipales, aunque encuadrados en zonas de penillanura o de regadío, como Talayuela (978,9 has.), Malpartida de Plasencia (802,2 has.) y Casatejada (1.307,7 has.).

Dentro de una localización geográfica determinada existe cierta heterogeneidad en su localización y desarrollo. A su emplazamiento normal en áreas montañosas podemos añadir una serie de excepciones muy marcadas, esto es, espacios muy localizados donde el rebollo forma espacios adehesados. Cabría citar las Sierras de Miravel y de la Perdiguera en el término municipal de Miravel, enclavados en el denominado Arco de Cañaveral en el 
centro de la provincia de Cáceres. Espacio adehesado que se sitúa alrededor de los $500 \mathrm{~m}$. de altura, muy por debajo del desarrollo altitudinal teórico de la especie, pero que por las características locales de orografía y climatología permite el desarrollo de esta especie.

Otro buen ejemplo de espacios adehesados con rebollar lo tenemos en el término de Talayuela (Campo Arañuelo) donde la altitud media es de $300 \mathrm{~m}$. Altura excesivamente baja para su desarrollo, pero debido al efecto de pantalla orográfica que supone la Sierra de Gredos al norte, le supone recibir unos aportes pluviométricos anuales considerables e, incluso, desarrollarse sobre suelos arenoso-limosos.

\section{Niveles de localización del rebollar en Extremadura}

Podemos encontrar cuatro niveles para la localización y diferenciación del rebollo en Extremadura, atendiendo al siguiente esquema:

a) Espacios con características físicas aptas para el desarrollo del rebollar y con superficies de rebollar.

b) Espacios con características óptimas para su desarrollo y sin superficies de rebollo.

c) Espacios sin características físicas idóneas para su desarrollo y con superficies de rebollar.

d) Espacios sin características físicas adecuadas y sin superficies de rebollo.

Dentro del primer grupo hemos de citar los espacios boscosos de rebollar, unido al castañar (Castanea sativa) en muchas ocasiones, de las zonas montañosas de VilluercasIbores (Sierra del Águila, 995 m. de altura; Sierra de Guadalupe, hasta los 1.000 m.,...); de la Sierra de Gredos (desde los 500 m. en los municipios del valle del río Tiétar hasta los $1.500 \mathrm{~m}$., donde da paso a los pastos de alta montaña).

Igualmente sucede en los términos ligados al río Jerte (tras la Sierra y El Valle) donde las superficies de rebollar comienzan a desarrollarse en los valles con masas abiertas para dar paso a zonas más densas de rebollo en las primeras pendientes y en las umbrías de las gargantas que han excavado los ríos y, por último, dan paso a los pastos de alta montaña por encima de los $800 \mathrm{~m}$. de altitud (GURRÍA, 1985).

Quedaría el sur de Badajoz (Sierra Morena extremeña) donde el rebollar tiene un desarrollo, eso sí un tanto irregular, cerca de los $1.000 \mathrm{~m}$. en los alrededores del monasterio de Tentudía (1.104 m.) en el término municipal de Calera de León. Esa localización en altura, más elevada que en el norte de Cáceres, implica necesariamente una serie de características climáticas determinadas y a su desarrollo en la vertiente norte de la Sierra Morena extremeña.

El segundo grupo define a unas áreas que han estado sometidas a una intensa deforestación del rebollar tanto para la obtención de maderas y leñas como para la optimización y consecución de pastizales para el ganado, al mismo tiempo también se han visto afectadas por políticas forestales de repoblación inadecuadas y por incendios posteriores.

Como ya mencionamos anteriormente, estos espacios están ligados fundamentalmente a las zonas de Las Hurdes y Sierra de Gata en el norte de la provincia de Cáceres.

Un tercer grupo queda conformado por aquellas áreas que, teóricamente, no cuentan con las características físicas adecuadas para el desarrollo del rebollo pero que, en ciertos casos, tienen superficies de rebollar. Estos espacios: a) no cumplen con la altura media desde la cual comienza a desarrollarse la especie (suelen situarse en estas zonas por debajo de los $700 \mathrm{~m}$. de altura); b) no se asientan sobre suelos pardos húmedos o subhúmedos; c) suelen tener temperaturas medias anuales más elevadas que los espacios con verdadera 
vocación de rebollar; d) son, por lo general, espacios peniaplanados o, incluso, llanos, pero con pendientes suaves en todo caso.

Los factores esenciales para que el rebollo se desarrolle en estos espacios son esencialmente dos:

- Elevadas precipitaciones anuales que se traducen en una humedad edáfica y ambiental óptima para el desarrollo del Quercus pyrenaica Willd. Esto supone una humedad constante a nivel de vuelo o copa (humedad ambiental) y a nivel de suelo y tronco (humedad edáfica). Estas abundantes precipitaciones se deben, en todo caso, al efecto de pantalla orográfica que ejercen los relieves montañosos del norte de Cáceres, que favorecen la extensión de la isoyeta de los $800 \mathrm{~mm}$. hasta el río Tajo.

- Existencia de fuertes inversiones térmicas en los meses invernales en zonas de valles y hondonadas de montaña, lo cual supone la introducción de fuertes cambios en la distribución de las especies altitudinalmente.

En estos casos, el rebollo suele ocupar las zonas más bajas de los valles, mucho más frías a nivel ambiental y del suelo que las laderas más elevadas donde, de este modo, se producen inversiones en la cliserie y podemos encontrar chaparros y pequeñas encinas achaparradas debido a que cuentan con temperaturas más suaves para su aparición y desarrollo.

El factor de la inversión térmica de irradiación, producida por el enfriamiento de los suelos continentales en las largas noches, con cielo despejado y viento encalmado, ocurren dentro de la misma masa de aire y son inducidas por enfriamiento de aire (GARCÍA DE PEDRAZA, 1989), junto a la importancia de las pendientes, la dirección de los vientos (valle-montaña o viceversa) supone que ante un aire muy saturado se produzcan también las nieblas de irradiación. Estos fenómenos contribuyen de manera decisiva a una distribución irregular de la vegetación manteniendo una correlación inversa de las especies y la altura.

Estas irregularidades en la distribución del rebollar se manifiestan de manera muy clara en casi toda la zona interior de Villuercas-Ibores y sistemas montañosos del norte de la región.

Tanto la floración y brote de estas especies en los meses primaverales dependen, en todo caso, de los retrocesos bruscos al frío invernal y de la persistencia de situaciones de heladas de irradiación debido a situaciones anticiclónicas de aire seco con noches de cielos despejados, suelo frío y viento encalmado. Tanto uno como factor climático son causa de esa distribución del rebollar en áreas no muy óptimas para su desarrollo.

Un último grupo, estaría formado por aquellos espacios sin condiciones edafoclimáticas idóneas para el desarrollo del rebollar y, evidentemente, sin rebollar. Serían espacios como la penillanura arbolada de encinas, los pastizales, las vegas de regadío, el secano intensivo y el olivar y viñedo (Mapa de localización).

\section{Características climáticas del rebollar en Extremadura}

Dentro de la zona de montaña en Extremadura, apta físicamente a la presencia del rebollo, hemos seleccionado tres observatorios meteorológicos: Cabeza la Vaca $\left(38^{\circ} 05^{\prime} \mathrm{N}\right.$ $6^{\circ} 25^{\prime} \mathrm{W}$, situado en Sierra Morena, en el sur de la provincia de Badajoz, a $759 \mathrm{~m}$. de altura), Guadalupe $\left(39^{\circ} 27^{\prime} \mathrm{N}-5^{\circ} 19^{\prime} \mathrm{W}\right.$, ubicado en el corazón de las Villuercas y a una altura de 640 m.) y Hervás $\left(40^{\circ} 16^{\prime} \mathrm{N}-5^{\circ} 51^{\prime} \mathrm{W}\right.$, emplazado en la Sierra de Gredos y a $688 \mathrm{~m}$. de altitud). 
Hemos tratado de seleccionar estos observatorios tanto por su serie de observación, bastante amplia en el tiempo (1961-1990), como por su distribución espacial heterogénea en la montaña extremeña.

Las temperaturas medias anuales en las que se mueven estos espacios oscilan entre los $15,49^{\circ} \mathrm{C}$ de Hervás y los $16,15^{\circ} \mathrm{C}$ de Guadalupe.

En líneas generales podemos establecer el intervalo de $15^{\circ}$ a $16^{\circ} \mathrm{C}$ para la media anual de medias.

La oscilación térmica anual se establece entre los $17^{\circ}$ y los $18^{\circ} \mathrm{C}$; la menor amplitud térmica anual se registra en Cabeza la Vaca con $17,21^{\circ} \mathrm{C}$ y la mayor oscilación la tiene el observatorio de Guadalupe con cerca de $18^{\circ} \mathrm{C}$.

En cualquier caso, presenta los siguientes registros medios mensuales:

Cuadro I

TEMPERATURAS MEDIAS MENSUALES

\begin{tabular}{|lccc|}
\hline MESES & CABEZA VACA & GUADALUPE & HERVÁS \\
\hline Enero & 7,40 & 7,72 & 7,15 \\
Febrero & 8,48 & 9,15 & 8,21 \\
Marzo & 10,88 & 11,79 & 11,41 \\
Abril & 13,66 & 13,78 & 13,17 \\
Mayo & 16,96 & 17,76 & 17,15 \\
Junio & 21,67 & 22,65 & 22,57 \\
Julio & 24,53 & 25,71 & 25,00 \\
Agosto & 24,61 & 25,49 & 24,53 \\
Septiembre & 21,73 & 22,67 & 21,51 \\
Octubre & 16,93 & 17,34 & 16,21 \\
Noviembre & 11,29 & 11,73 & 10,97 \\
Diciembre & 8,17 & 8,08 & 8,01 \\
MEDIA & 15,52 & 16,15 & 15,49 \\
\hline
\end{tabular}

Elaboración propia a partir del I.N.M.

Como podemos observar las diferencias son mínimas entre los observatorios seleccionados, prueba de ello es la desviación tan escasa que tienen las temperaturas medias anuales.

Desde el mes de octubre hasta mayo se registran temperaturas mínimas inferiores a $7^{\circ} \mathrm{C}$, lo cual supone un riesgo potencial de heladas muy elevado, casi ocho meses, puesto que las mínimas absolutas deben ser inferiores a $0^{\circ} \mathrm{C}$.

La situación de estos observatorios en zonas de montaña significa, entre otras cosas, que las máximas medias estivales sean atenuadas, en ningún caso se superan $l o s 40^{\circ} \mathrm{C} \mathrm{y}$, en invierno, como consecuencia de las inversiones térmicas y de la propia situación de algunos observatorios, no sean tan bajas.

Las características pluviométricas de los espacios montañosos de rebollar suponen, en cualquier caso, precipitaciones medias anuales superiores a $800 \mathrm{~mm}$. Las estaciones meteorológicas seleccionadas para tal efecto muestran los siguientes valores: 
Cuadro II

PRECIPITACIONES MEDIAS MENSUALES

\begin{tabular}{|lccc|}
\hline MESES & CABEZA VACA & GUADALUPE & HERVÁS \\
\hline Enero & 129,61 & 124,48 & 122,80 \\
Febrero & 113,58 & 113,28 & 132,06 \\
Marzo & 77,50 & 68,62 & 88,79 \\
Abril & 79,12 & 82,61 & 93,34 \\
Mayo & 51,81 & 52,13 & 78,03 \\
Junio & 39,72 & 36,34 & 49,54 \\
Julio & 5,78 & 8,47 & 15,27 \\
Agosto & 4,69 & 7,63 & 11,54 \\
Septiembre & 32,44 & 34,55 & 54,91 \\
Octubre & 85,75 & 77,64 & 93,41 \\
Noviembre & 117,60 & 131,46 & 145,66 \\
Diciembre & 113,96 & 147,33 & 125,44 \\
TOTAL & 851,56 & 884,54 & $1.010,79$ \\
\hline
\end{tabular}

Elaboración propia a partir del I.N.M.

A estas elevadas precipitaciones le acompaña un importante número de días de precipitación al año. Se pueden cifrar en más de 75 días de precipitación apreciable al año. Utilizando las precipitaciones, las temperaturas medias, el índice de calor, la e.t.p. sin corregir (en mm.) y el índice de iluminación calculamos la ficha termohídrica correspondiente, obteniendo unos valores bajos de la E.T.P. (823,62 mm./año) y, claro está, más bajo aún será la evapotranspiración actual que se cifra en 525,92 mm./año. (Estos datos se refieren al observatorio de Hervás pero pueden ser extrapolables al resto de ellos).

Dadas unas condiciones térmicas y pluviométricas determinadas, la reserva de agua en el suelo va a ser eficiente durante 9 meses (octubre a junio), quedando solamente los meses de julio a septiembre como meses con déficits hídricos importantes y, en definitiva, con un déficit de agua que se cifra en $297,70 \mathrm{~mm}$. Un hecho esencial porque es el momento en que esta especie se encuentra en un importante y casi óptimo desarrollo vegetativo. A todo ello hemos de unirle, los meses de diciembre a febrero en los que la reserva del suelo es excedentaria; esta saturación puede provocar procesos erosivos causados por la escorrentía superficial.

\section{Conclusiones}

A pesar del gran interés que despierta desde diferentes puntos de vista las superficies arboladas de rebollar, su situación actual dista bastante de ser plenamente satisfactoria. La degradación de la vegetación climácica se ha producido de muy diversas formas, entre ellas caben destacar las siguientes:

- Incendios provocados.

- Cortas indiscriminadas del arbolado.

- Repoblación con especies alóctonas.

Como se puede comprobar, en todas ellas el factor antrópico está presente con todo lo que ella representa. Cualesquiera que sea el proceso degradativo en cuestión, las etapas de 


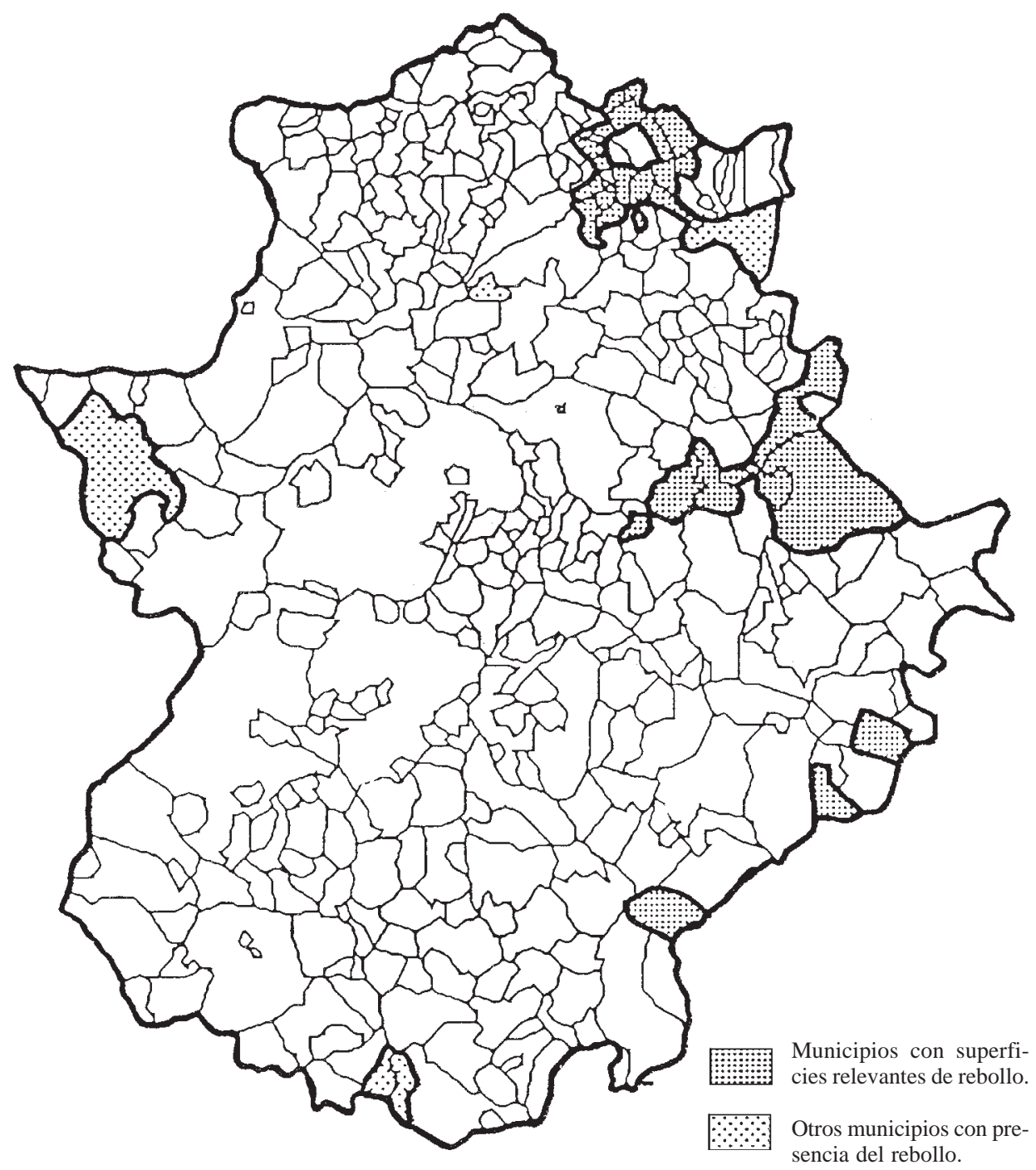

Fuente: Elaboración propia a partir de ALVARADO CORRALES, E. (1983): El sector forestal en Extremadura, Ecología y Economía. Institución Cultural «El Brocense». Cáceres.

degradación son muy claras: una reducción de la biomasa vegetal protectora, y un menor aporte superficial de nutrientes (MESÓN GARCÍA et al., 1985). Estos aspectos conducen, en definitiva, hacia una evidente pérdida de fertilidad debido al menor contenido de nutrientes del suelo.

Entre otros efectos sobre el suelo pueden concurrir los siguientes: ..., deterioro de la estructura (que se hace cada vez más inestable al perder cationes y sobre todo calcio), acidificación superficial (que implica un más fácil arrastre de coloides), aumento de la 
compacidad, reducción de la permeabilidad, reducción de la capacidad de retención del agua (estas tres como consecuencia del deterioro de la estructura), formación de un horizonte B cada vez de más difícil habitabilidad para las raíces, etc. (MESÓN GARCÍA et al., 1985).

La pérdida del matorral alto y denso que acompaña a la propia orla del bosque de rebollos (Ruscus aculeatus, Daphne gnidium, Viburnum tinus,...) supone perder una excelente pantalla protectora del suelo frente a los procesos erosivos, así como una pérdida de mineralización de los mismos. Cuando ese sotobosque queda compuesto por matorral de brezal o sus propios renuevos, la protección física es mucho menos eficaz y, consecuentemente, el suelo sufre más esos efectos.

Podemos significar que la existencia del rebollar en Extremadura pese a tener una gran extensión geográfica (Cordillera Mariánica o Sierra Morena, vertiente meridional de Gredos — Sector Verense-, desde Candeleda —Ávila - llega hasta las sierras de Béjar y Peña de Francia y también en Villuercas en la Oretana Central, por la Oretana Occidental llega hasta las sierras de San Mamede y Montánchez), no ocupa demasiada extensión superficial. En Extremadura sólo hay una superficie de rebollo de unas 33.477 has., un $2,7 \%$ del total de las quercíneas; dentro de éstas es la encina, con más de 1 millón de has., la especie más representativa. En conjunto la región extremeña cuenta con 1.211 .883 has. de quercíneas, aproximadamente un $29 \%$ del total de la superficie regional.

Por tanto, urgen medidas inmediatas para la conservación del rebollo en estas áreas y, además, será necesaria la repoblación de esta especie en aquellas áreas de montaña (Sierra de Gata y Las Hurdes) en las que ya estuvo presente. El apoyo de la Administración Pública camina, de momento, por unas directrices idóneas, la puesta en marcha hace dos años del Plan de Forestación de la Junta de Extremadura (Decreto 95/1993, de 20 de julio) debe ser el punto de partida para el desarrollo del bosque en estas áreas tan devastadas en el tiempo.

\section{Bibliografía}

ALBENTOSA SÁNCHEZ, L.M. (1982): «Introducción al estudio sistemático de la degradación del medio ambiente en la provincia de Tarragona». Tarraco, $\mathrm{n}^{\circ}$ 3. Dpto. de Geografía de la Universidad de Barcelona, pp. 105-139.

ALVARADO CORRALES, E. (1983): El sector forestal en Extremadura. Ecología y Economía. Institución Cultural «El Brocense». Cáceres.

GARCÍA DE PEDRAZA, L. (1989): «Dos tipos de adversidades meteorológicas: La nevada y el granizo». Norba, VI-VII. Revista de Geografía. Universidad de Extremadura. Cáceres, pp. 21-30.

GURRÍA GASCÓN, J.L. (1985): El paisaje de montaña en Extremadura. (Delimitación, Economía y Población). Universidad de Extremadura, Cáceres.

LADERO ÁLVAREZ, M. (1987): «La España Luso-Extremadurense», en La vegetación de España, V.V.A.A. Universidad de Alcalá de Henares, pp. 453-488.

LECO BERROCAL, F. (1995): Ecosistema dehesa: Aproximación metodológica a su delimitación, ordenación y transformación. (Tesis Doctoral). Dpto. de Geografía y Ordenación del Territorio. Universidad de Extremadura, Cáceres.

MESÓN GARCÍA, Ma .L. et al. (1985): «Vegetación forestal y degradación de los bosques de Quercus pyrenacia Willd en España». M.A.P.A.-I.N.I.A. Serie: Recursos Naturales, $\mathrm{n}^{\circ}$ 41. Madrid. 